\title{
Paper 1: "Cement as a thermoelectric material," [J. Mater. Res. 15, 2844-2848 (2000)] \\ Paper 2: "Rectifying and thermocouple junctions based on Portland cement," [J. Mater. Res. 16, 1989-1993 (2001)]
}

\author{
Sihai Wen and D.D.L. Chung \\ Composite Materials Research Laboratory, University at Buffalo, State University of New York, \\ Buffalo, New York 14260-4400
}

(Received 23 January 2004; accepted 6 February 2004)

In the two papers listed above, the conversion of the Seebeck coefficient (relative to copper) to the absolute thermoelectric power was done by using the wrong sign of the absolute thermoelectric power of copper $\left(2.34 \mu \mathrm{V} /{ }^{\circ} \mathrm{C}\right)$. The corrected tables are shown below for both papers.

The correction means that plain cement paste is slightly p-type rather than slightly n-type. In addition, it means that cement pastes with carbon fibers are more p-type and those with steel fibers are less n-type than reported.

Note in Table III of Paper 2 that all cement pastes are p-type except for paste (ii). Note in Table IV of Paper 2 that all cement junctions are pn-junctions (rather than some being $\mathrm{nn}^{+}$-junctions).

TABLE IV (Paper 1). Volume electrical resistivity, Seebeck coefficient $\left(\mu \mathrm{V} /{ }^{\circ} \mathrm{C}\right)$ with copper as the reference, and the absolute thermoelectric power $\left(\mu \mathrm{V} /{ }^{\circ} \mathrm{C}\right)$ of various cement pastes with steel fibers $\left(\mathrm{S}_{\mathrm{f}}\right)$ or carbon fibers $\left(\mathrm{C}_{\mathrm{f}}\right)$.

\begin{tabular}{|c|c|c|c|c|c|c|}
\hline \multirow[b]{2}{*}{ Cement paste } & \multirow[b]{2}{*}{$\begin{array}{c}\text { Volume } \\
\text { fraction fibers }\end{array}$} & \multirow[b]{2}{*}{$\begin{array}{l}\text { Resistivity } \\
(\Omega \cdot \mathrm{cm})\end{array}$} & \multicolumn{2}{|c|}{ Heating } & \multicolumn{2}{|c|}{ Cooling } \\
\hline & & & $\begin{array}{l}\text { Seebeck } \\
\text { coefficient }\end{array}$ & $\begin{array}{l}\text { Absolute } \\
\text { thermoelectric power }\end{array}$ & $\begin{array}{l}\text { Seebeck } \\
\text { coefficient }\end{array}$ & $\begin{array}{l}\text { Absolute } \\
\text { thermoelectric power }\end{array}$ \\
\hline Plain & 0 & $\ldots$ & $+0.35 \pm 0.03$ & $+2.69 \pm 0.03$ & $+0.38 \pm 0.05$ & $+2.72 \pm 0.05$ \\
\hline SF & 0 & $\ldots$ & $+0.31 \pm 0.02$ & $+2.65 \pm 0.02$ & $+0.36 \pm 0.03$ & $+2.70 \pm 0.03$ \\
\hline $\mathrm{L}$ & 0 & $\cdots$ & $+0.28 \pm 0.02$ & $+2.62 \pm 0.02$ & $+0.30 \pm 0.02$ & $+2.64 \pm 0.02$ \\
\hline $\mathrm{S}_{\mathrm{f}}\left(0.5^{*}\right)$ & $0.10 \%$ & $(7.8 \pm 0.5) \times 10^{4}$ & $-51.0 \pm 4.8$ & $-48.7 \pm 4.8$ & $-45.3 \pm 4.4$ & $-43.0 \pm 4.4$ \\
\hline $\mathrm{S}_{\mathrm{f}}\left(1.0^{*}\right)$ & $0.20 \%$ & $(4.8 \pm 0.4) \times 10^{4}$ & $-56.8 \pm 5.2$ & $-54.5 \pm 5.2$ & $-53.7 \pm 4.9$ & $-51.4 \pm 4.9$ \\
\hline $\mathrm{S}_{\mathrm{f}}\left(0.5^{*}\right)+\mathrm{SF}$ & $0.10 \%$ & $(5.6 \pm 0.5) \times 10^{4}$ & $-54.8 \pm 3.9$ & $-52.5 \pm 3.9$ & $-52.9 \pm 4.1$ & $-50.6 \pm 4.1$ \\
\hline $\mathrm{S}_{\mathrm{f}}\left(1.0^{*}\right)+\mathrm{SF}$ & $0.20 \%$ & $(3.2 \pm 0.3)+10^{4}$ & $-66.2 \pm 4.5$ & $-63.9 \pm 4.5$ & $-65.6 \pm 4.4$ & $-63.3 \pm 4.4$ \\
\hline $\mathrm{S}_{\mathrm{f}}\left(0.5^{*}\right)+\mathrm{L}$ & $0.085 \%$ & $(1.4 \pm 0.1) \times 10^{5}$ & $-48.1 \pm 3.2$ & $-45.8 \pm 3.2$ & $-45.4 \pm 2.9$ & $-43.1 \pm 2.9$ \\
\hline $\mathrm{S}_{\mathrm{f}}\left(1.0^{*}\right)+\mathrm{L}$ & $0.17 \%$ & $(1.1 \pm 0.1) \times 10^{5}$ & $-55.4 \pm 5.0$ & $-53.1 \pm 5.0$ & $-54.2 \pm 4.5$ & $-51.9 \pm 4.5$ \\
\hline $\mathrm{C}_{\mathrm{f}}\left(0.5^{*}\right)+\mathrm{SF}$ & $0.48 \%$ & $(1.5 \pm 0.1) \times 10^{4}$ & $+1.45 \pm 0.09$ & $+3.79 \pm 0.09$ & $+1.45 \pm 0.09$ & $+3.79 \pm 0.09$ \\
\hline $\mathrm{C}_{\mathrm{f}}\left(1.0^{*}\right)+\mathrm{SF}$ & $0.95 \%$ & $(8.3 \pm 0.5)+10^{2}$ & $+2.82 \pm 0.11$ & $+5.16 \pm 0.11$ & $+2.82 \pm 0.11$ & $+5.16 \pm 0.11$ \\
\hline$C_{f}\left(1.5^{*}\right)+S F$ & $1.44 \%$ & $\cdots$ & $+3.10 \pm 0.14$ & $+5.44 \pm 0.14$ & $+3.10 \pm 0.14$ & $+5.44 \pm 0.14$ \\
\hline $\mathrm{C}_{\mathrm{f}}\left(0.5^{*}\right)+\mathrm{L}$ & $0.41 \%$ & $(9.7 \pm 0.6) \times 10^{4}$ & $+1.20 \pm 0.05$ & $+3.54 \pm 0.05$ & $+1.20 \pm 0.05$ & $+3.54 \pm 0.05$ \\
\hline $\mathrm{C}_{\mathrm{f}}(1.0 *)+\mathrm{L}$ & $0.82 \%$ & $(1.8 \pm 0.2)+10^{3}$ & $+2.10 \pm 0.08$ & $+4.44 \pm 0.08$ & $+2.10 \pm 0.08$ & $+4.44 \pm 0.08$ \\
\hline
\end{tabular}

*\% by mass of cement.

SF: silica fume.

L: latex.

TABLE III (Paper 2). Absolute thermoelectric power $\left(\mu \mathrm{V} /{ }^{\circ} \mathrm{C}\right)$.

\begin{tabular}{lcllr}
\hline \hline \multicolumn{1}{c}{$\begin{array}{c}\text { Cement } \\
\text { paste }\end{array}$} & $\begin{array}{c}\text { Volume } \\
\text { fraction fibers }\end{array}$ & \multicolumn{1}{c}{$\mu \mathrm{V} /{ }^{\circ} \mathrm{C}$} & \multicolumn{1}{c}{ Type } & Ref. \\
\hline (i) Plain & 0 & $+2.69 \pm 0.03$ & Weakly p & 1 \\
(ii) $\mathrm{S}_{\mathrm{f}}\left(0.5^{*}\right)$ & $0.10 \%$ & $-48.7 \pm 4.8$ & Strongly n & 5 \\
(iii) $\mathrm{C}_{\mathrm{f}}\left(0.5^{*}\right)+\mathrm{SF}$ & $0.48 \%$ & $+3.79 \pm 0.09$ & Weakly p & 1 \\
(iv) $\mathrm{C}_{\mathrm{f}}\left(1.0^{*}\right)+\mathrm{SF}$ & $0.95 \%$ & $+5.16 \pm 0.11$ & $\mathrm{p}$ & 1 \\
(v) $\mathrm{C}_{\mathrm{f}}\left(0.5^{*}\right)+\mathrm{L}$ & $0.41 \%$ & $+3.54 \pm 0.05$ & Weakly p & 1 \\
\hline \hline
\end{tabular}

TABLE IV (Paper 2). Cement junctions.

\begin{tabular}{|c|c|c|c|c|}
\hline \multirow[b]{2}{*}{ Junction } & \multirow{2}{*}{$\begin{array}{l}\text { Pastes } \\
\text { involved }\end{array}$} & \multirow{2}{*}{$\begin{array}{l}\text { Junction } \\
\text { type }\end{array}$} & \multicolumn{2}{|c|}{$\begin{array}{l}\text { Thermocouple sensitivity } \\
\left(\mu \mathrm{V} /{ }^{\circ} \mathrm{C}\right)\end{array}$} \\
\hline & & & Heating & Cooling \\
\hline (a) & (iv) and (ii) & pn & $70 \pm 7$ & $70 \pm 7$ \\
\hline (b) & (iii) and (ii) & pn & $65 \pm 5$ & $65 \pm 6$ \\
\hline (c) & (v) and (ii) & pn & $59 \pm 7$ & $58 \pm 5$ \\
\hline
\end{tabular}

Note: $\mathrm{SF}=$ silica fume; $\mathrm{L}=$ latex.

DOI: $10.1557 / J M R .2004 .0169$ 